SUPPORTING INFORMATION FOR

\title{
Element-Hydrogen Bond Activations at Cationic Platinum Centers to Produce Silylene, Germylene, Stannylene, and Stibido Complexes
}

\author{
Rory Waterman, ${ }^{\ddagger}$ Rex C. Handford, ${ }^{\sharp}$ and T. Don Tilley,,* \\ ${ }^{\ddagger}$ Department of Chemistry, University of Vermont, Burlington, Vermont 05405 \\ ${ }^{*}$ Department of Chemistry, University of California, Berkeley, California 94720-1460
}

\section{TABLE OF CONTENTS}

S2: Details of crystallography

S3: Table S1. Crystal data and structure refinement for 14

S4: Details of calculations

S5: ${ }^{1} \mathrm{H}$ NMR spectra of new compounds

S16: References 


\section{DETAILS OF CRYSTALLOGRAPHY}

\section{Data collection}

A yellow block of $[($ dippe $) \mathrm{Pt}(\mu-\mathrm{SbHMes})]\left[\mathrm{BAr}_{4}^{\mathrm{f}}\right]$ (11) was cut from a larger block under Paratone-N oil to minimize reaction with air. The crystal was removed from the oil using a plastic loop that also served to hold the crystal for data collection. The crystal was then mounted and centered on a Bruker SMART system. Rotation and still images showed diffractions to be sharp. Frames separated in reciprocal space were obtained and provided an orientation matrix and initial cell parameters. Final cell parameters were obtained from the full data set.

A "hemisphere" data set was obtained of reciprocal space to a resolution of $0.85 \AA$ using $0.3^{\circ}$ steps in $\omega$ using exposure times of $10 \mathrm{~s}$ for each frame. Data were collected at $145 \mathrm{~K}$. Integration of intensities and refinement of cell parameters were done using SAINT. Absorption corrections were applied using psi-scans and were small due to low absorption and equidimensional shape. Observation of the crystal after data collection showed no signs of decomposition.

\section{Structure solution and refinement}

The structure of 11 was solved by intrinsic phasing using the ShelXT ${ }^{1}$ and refined using the ShelXL ${ }^{2}$ software packages in the OLEX2 interace. ${ }^{3}$ Hydrogen atoms were added at calculated positions and refined isotropically. A relatively large residual electron density peak was located approximately along the $\mathrm{Pt}-\mathrm{Sb}$ bond, which could not be modeled as an atom. A residual electron density peak was assigned to the $\mathrm{H}$ atom of the $\mathrm{Sb}-\mathrm{H}$ linkage, but its position could not be reliably refined. There was mild disorder of the $\mathrm{CF}_{3}$ substituents of the $\left[\mathrm{BAr}_{4}^{\mathrm{f}}\right]^{-}$anion; ISOR, RIGU, and DELU commands were used on some atoms of the anion to maintain reasonable ADPs.

$$
\begin{array}{ll}
\mathrm{R}_{\text {int }}=\Sigma\left|\mathrm{F}_{\mathrm{o}}^{2}-\left\langle\mathrm{F}_{\mathrm{o}}^{2}\right\rangle\right| / \Sigma\left|\mathrm{F}_{\mathrm{o}}^{2}\right| & \mathrm{R}_{\mathrm{l}}=\Sigma|| \mathrm{F}_{\mathrm{o}}|-| \mathrm{F}_{\mathrm{c}}|| / \Sigma\left|\mathrm{F}_{\mathrm{o}}\right| \\
\mathrm{wR}_{2}=\left[\Sigma\left[\mathrm{w}\left(\mathrm{F}_{\mathrm{o}}^{2}-\mathrm{F}_{\mathrm{c}}^{2}\right)^{2}\right] / \Sigma\left[\mathrm{w}\left(\mathrm{F}_{\mathrm{o}}^{2}\right)^{2}\right]\right]^{1 / 2} & \text { where: } \mathrm{w}=\mathrm{q} / \sigma^{2}\left(\mathrm{~F}_{\mathrm{o}}^{2}\right)+(\mathrm{aP})^{2}+\mathrm{bP} ; \\
\mathrm{GoF}=\mathrm{S}=\left[\Sigma\left[\mathrm{w}\left(\mathrm{F}_{\mathrm{o}}^{2}-\mathrm{F}_{\mathrm{c}}^{2}\right)^{2}\right] /(\mathrm{n}-\mathrm{p})^{1 / 2}\right. & \mathrm{n}=\text { number of independent reflections; } \\
\quad \mathrm{p}=\text { number of parameters refined. } &
\end{array}
$$

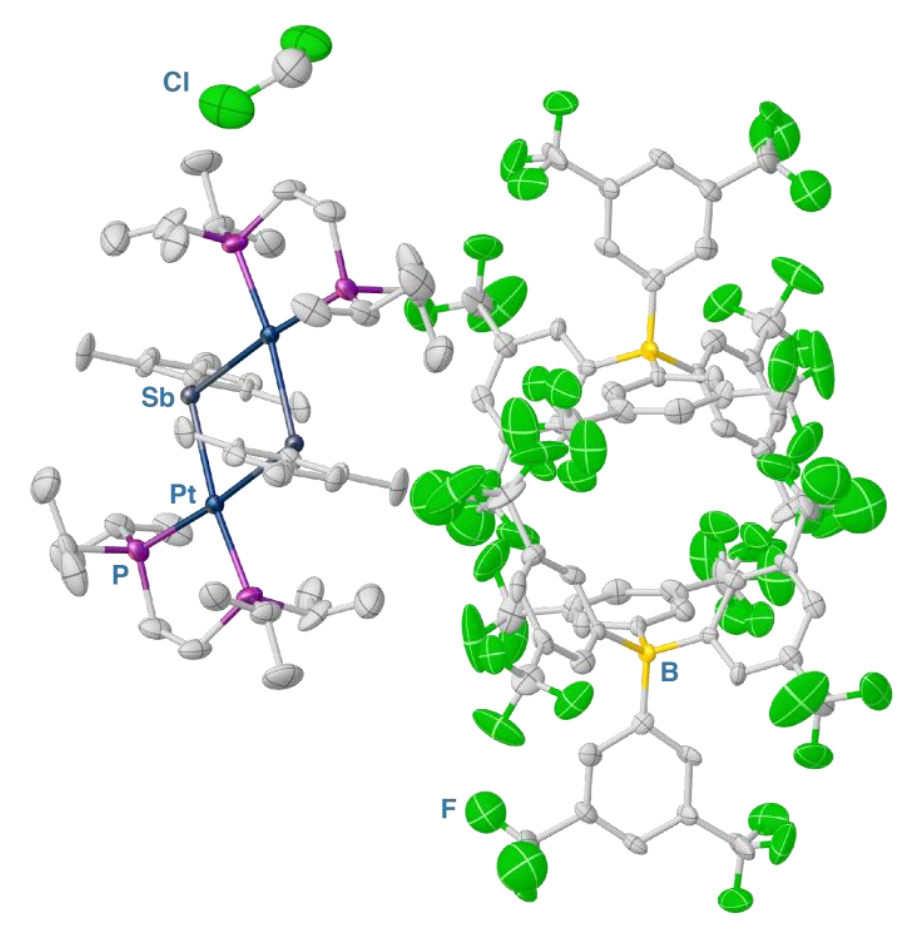

Figure S1. Full view of the solid-state molecular structure of $\mathbf{1 1}$, showing disorder of the $\left[\mathrm{BAr}_{4}^{\mathrm{f}}\right]^{-}$anions, and $\mathrm{CH}_{2} \mathrm{Cl}_{2}$ solvent molecule. Hydrogen atoms have been omitted for the sake of clarity. 
Table S1. Crystal data and structure refinement for 11 .

\begin{tabular}{|c|c|}
\hline Identification code & RW07_0m \\
\hline Empirical formula & $\mathrm{C}_{56} \mathrm{H}_{57} \mathrm{BCl}_{2} \mathrm{~F}_{24} \mathrm{P}_{2} \mathrm{PtSb}$ \\
\hline Formula weight & 1646.50 \\
\hline Temperature/K & 145 \\
\hline Crystal system & monoclinic \\
\hline Space group & $\mathrm{P} 2_{1} / \mathrm{c}$ \\
\hline $\mathrm{a} / \AA ̊$ & $15.439(3)$ \\
\hline $\mathrm{b} / \AA$ & $22.153(4)$ \\
\hline $\mathrm{c} / \AA ̊$ & $19.407(4)$ \\
\hline$\alpha /{ }^{\circ}$ & 90 \\
\hline$\beta /^{\circ}$ & $107.093(2)$ \\
\hline$\gamma /{ }^{\circ}$ & 90 \\
\hline Volume $/ \AA^{3}$ & $6344(2)$ \\
\hline $\mathrm{Z}$ & 4 \\
\hline$\rho_{\text {calcg }} / \mathrm{cm}^{3}$ & 1.724 \\
\hline$\mu / \mathrm{mm}^{-1}$ & 2.870 \\
\hline$F(000)$ & 3228.0 \\
\hline Crystal size $/ \mathrm{mm}^{3}$ & $1 \times 0.8 \times 0.6$ \\
\hline Radiation & $\operatorname{MoKa}(\lambda=0.71073 \AA)$ \\
\hline $2 \Theta$ range for data collection $/{ }^{\circ}$ & 5.728 to 52.198 \\
\hline Index ranges & $-19 \leq h \leq 11,-27 \leq k \leq 25,-23 \leq l \leq 22$ \\
\hline Reflections collected & 31652 \\
\hline Independent reflections & $12221\left[R_{\mathrm{int}}=0.0763, R_{\mathrm{sigma}}=0.1130\right]$ \\
\hline Data/restraints/parameters & $12221 / 84 / 822$ \\
\hline Goodness-of-fit on $F^{2}$ & 1.062 \\
\hline Final $R$ indexes $[\mathrm{I}>=2 \sigma(\mathrm{I})]$ & $R_{1}=0.0729, \mathrm{w} R_{2}=0.1771$ \\
\hline Final $R$ indexes [all data] & $R_{1}=0.1258, \mathrm{w} R_{2}=0.2045$ \\
\hline Largest diff. peak/hole / e $\AA^{-3}$ & $3.40 /-1.23$ \\
\hline
\end{tabular}




\section{DETAILS OF DFT CALCULATIONS}

The structures of $\left[(\mathrm{dmpe}) \mathrm{Pt}(\mathrm{H})=\mathrm{EPh}_{2}\right]^{+}\left(\mathrm{E}=\mathrm{Si}, \mathbf{1}^{*} ; \mathrm{E}=\mathrm{Ge}, \mathbf{8}^{*}, \mathrm{E}=\mathrm{Sn}, \mathbf{9}^{*}\right)$ were optimized using the $\mathrm{PBE}$ functional ${ }^{4}$ in Gaussian 16 (rev. A.03). ${ }^{5}$ For $\mathbf{1}^{*}, \mathbf{8}^{*}$, and $\mathbf{9}^{*}$, all $\mathrm{C}$ and $\mathrm{H}$ atoms (except for the hydride atom) were specified with def2-svp basis sets, ${ }^{6}$ the $\mathrm{P}$ atoms and hydride atom with the def2-svpd basis set, and the Pt atom with the def2-tzvpd basis set. ${ }^{7}$ The $\mathrm{Si}, \mathrm{Ge}$, and $\mathrm{Sn}$ atoms were specified with def2-svpd, def2-svpd, and def2-tzvpd basis sets, respectively. ${ }^{7}$ Effective core potentials (ECPs) for the Pt and Sn atoms were modeled using def2-tzvp ECPs. ${ }^{8,9}$ Density fitting of C, H, P, Si, and Ge atoms in these complexes was performed with the w06 basis, while the Pt and Sn atoms were fitted with their respective def2-tzvp Jbases. ${ }^{10}$ For all structures, the absence of negative frequencies verified that the optimized structures corresponded to true minima. Visualization of the computed molecular orbitals depicted in Figure 1 was performed using VMD. ${ }^{11}$

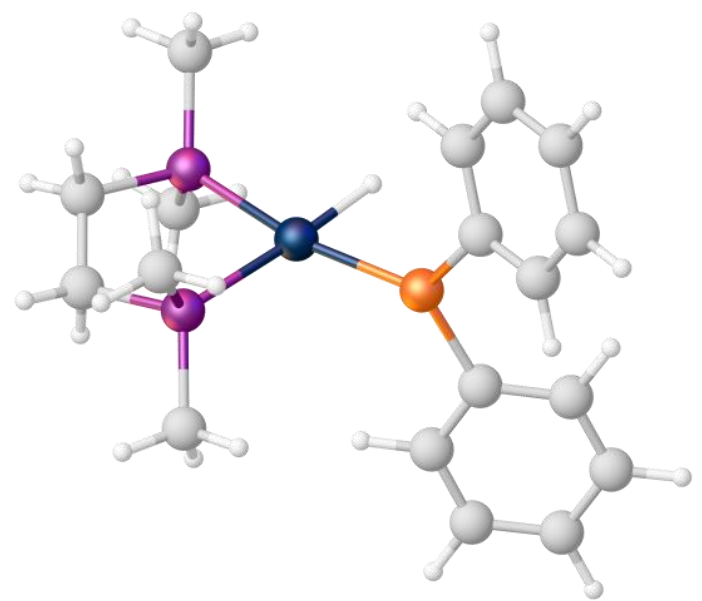

Figure S2. Optimized geometry of $\mathbf{1}^{*}$.

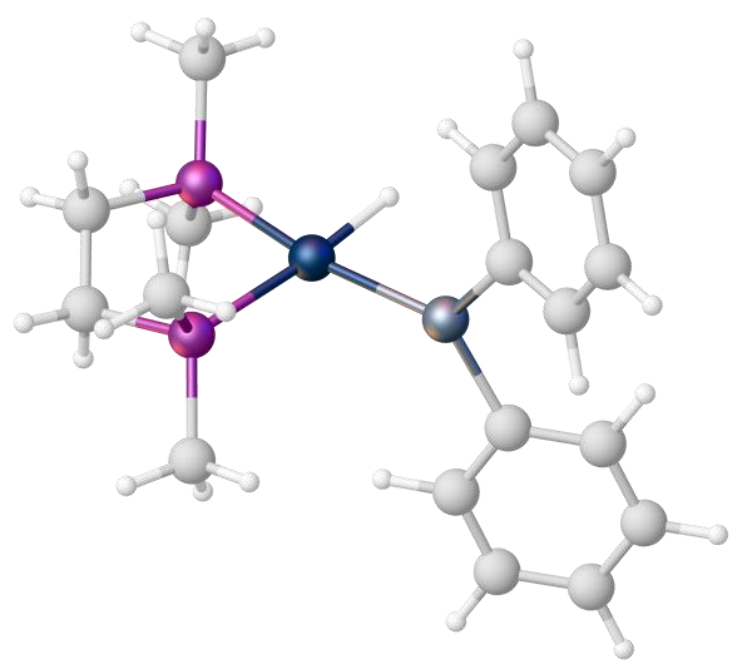

Figure S3. Optimized geometry of $\mathbf{8}^{*}$.

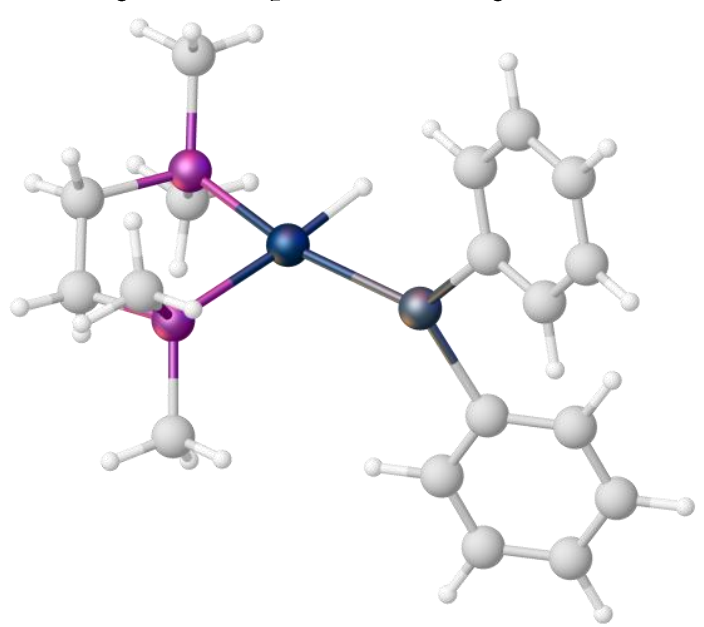

Figure S4. Optimized geometry of $\mathbf{9}^{*}$. 
${ }^{1} \mathrm{H}$ NMR SPECTRA OF NEW COMPOUNDS

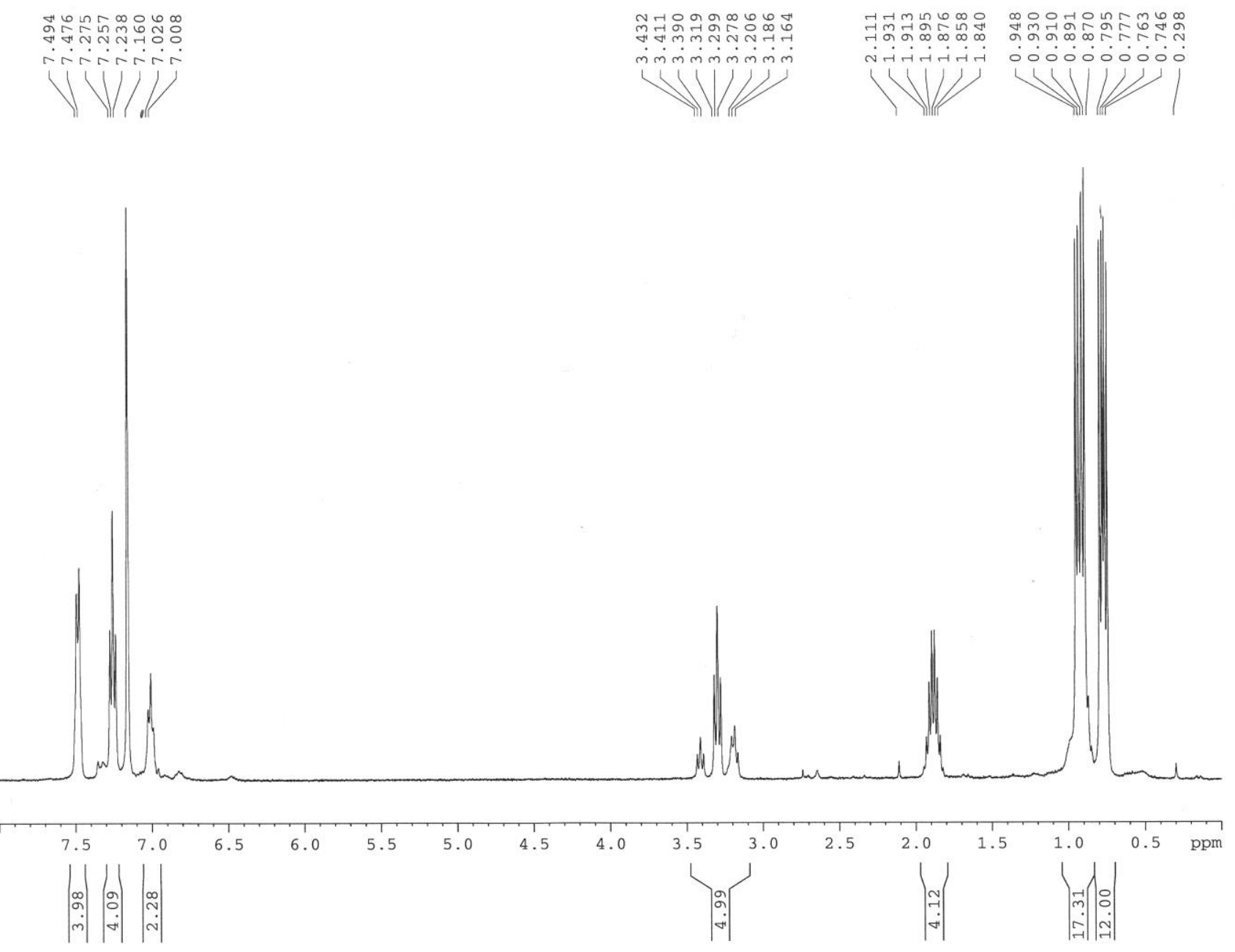

Figure S5. ${ }^{1} \mathrm{H}$ NMR (benzene- $d_{6}, 400 \mathrm{MHz}, 293 \mathrm{~K}$ ) spectrum of 2 . 

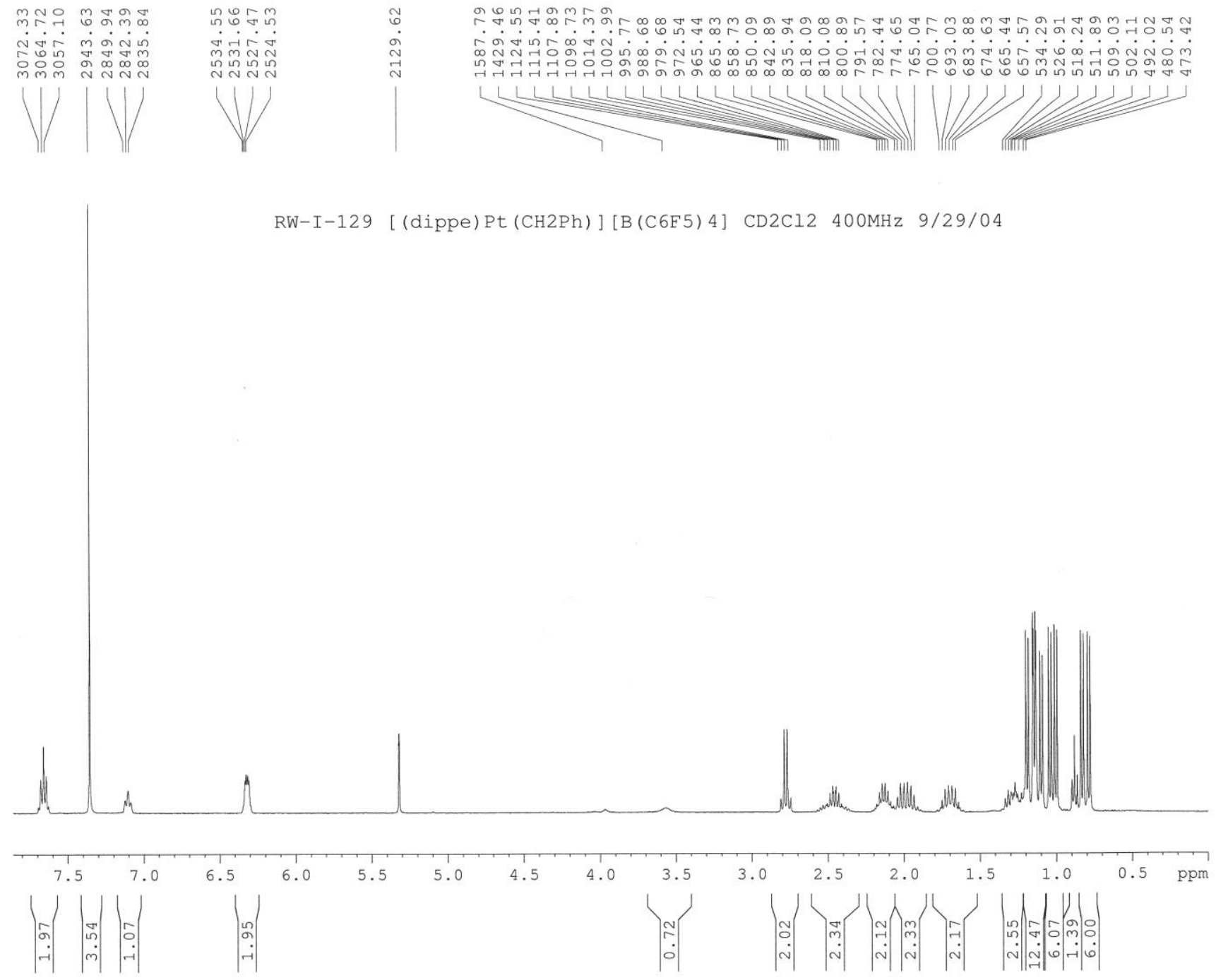

Figure S6. ${ }^{1} \mathrm{H} \mathrm{NMR}\left(\mathrm{CD}_{2} \mathrm{Cl}_{2}, 400 \mathrm{MHz}, 293 \mathrm{~K}\right)$ spectrum of 3. 


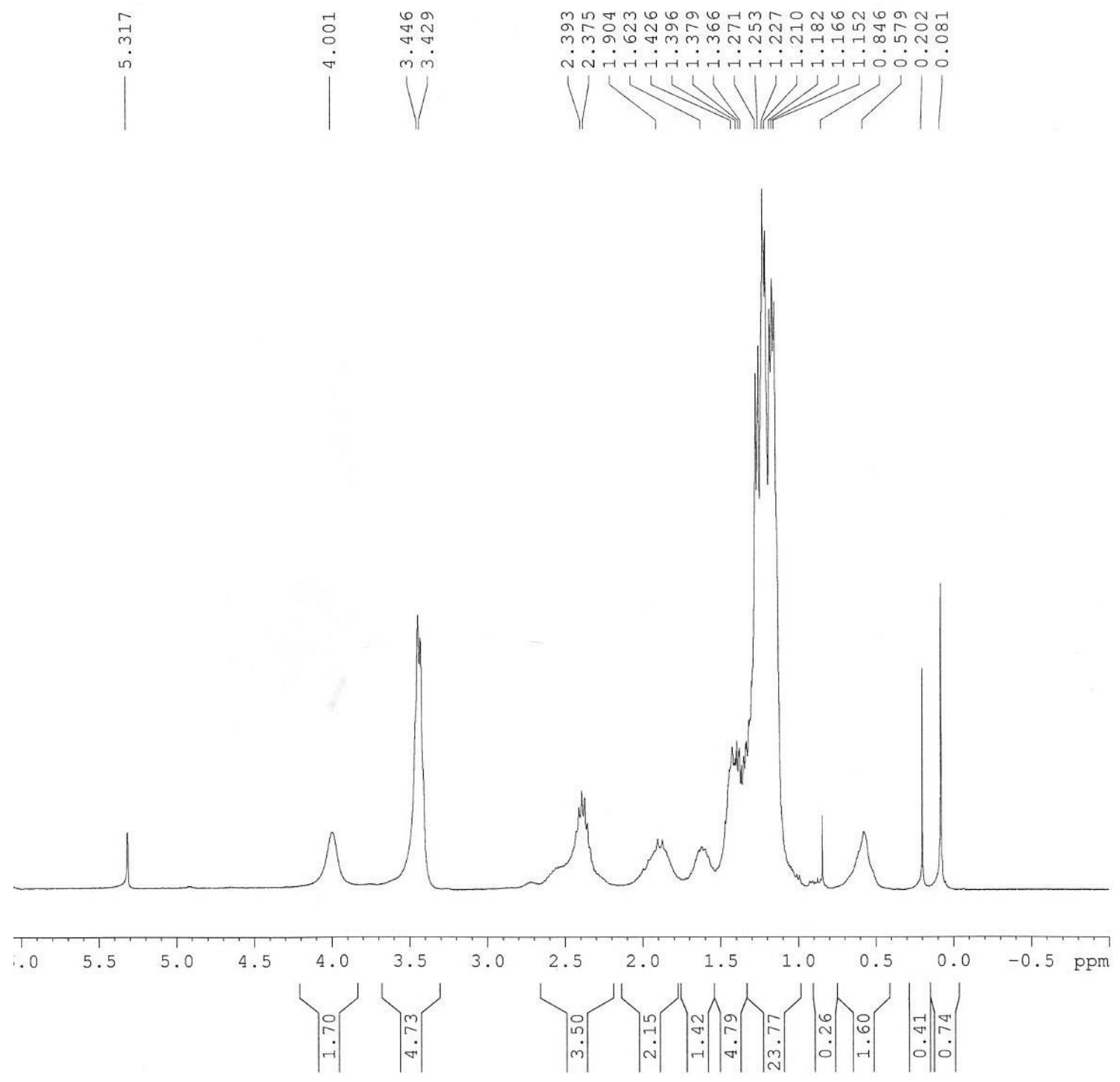

Figure S7. ${ }^{1} \mathrm{H} \mathrm{NMR}\left(\mathrm{CD}_{2} \mathrm{Cl}_{2}, 400 \mathrm{MHz}, 293 \mathrm{~K}\right)$ spectrum of $\mathbf{4}\left[\mathrm{B}\left(\mathrm{C}_{6} \mathrm{~F}_{5}\right)_{4}\right]$. 


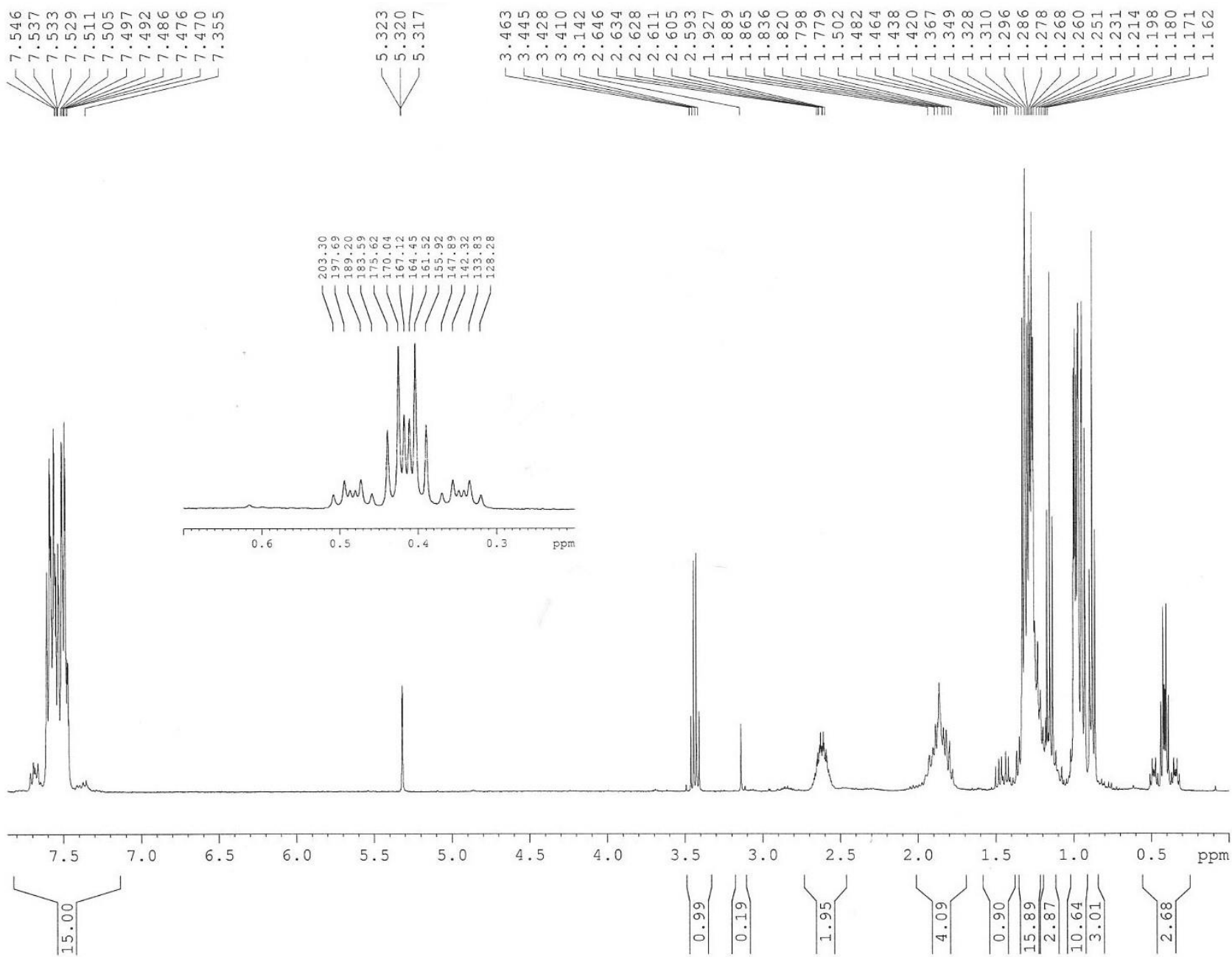

Figure S8. ${ }^{1} \mathrm{H} \mathrm{NMR}\left(\mathrm{CD}_{2} \mathrm{Cl}_{2}, 400 \mathrm{MHz}, 293 \mathrm{~K}\right)$ spectrum of $\mathbf{5}$. 

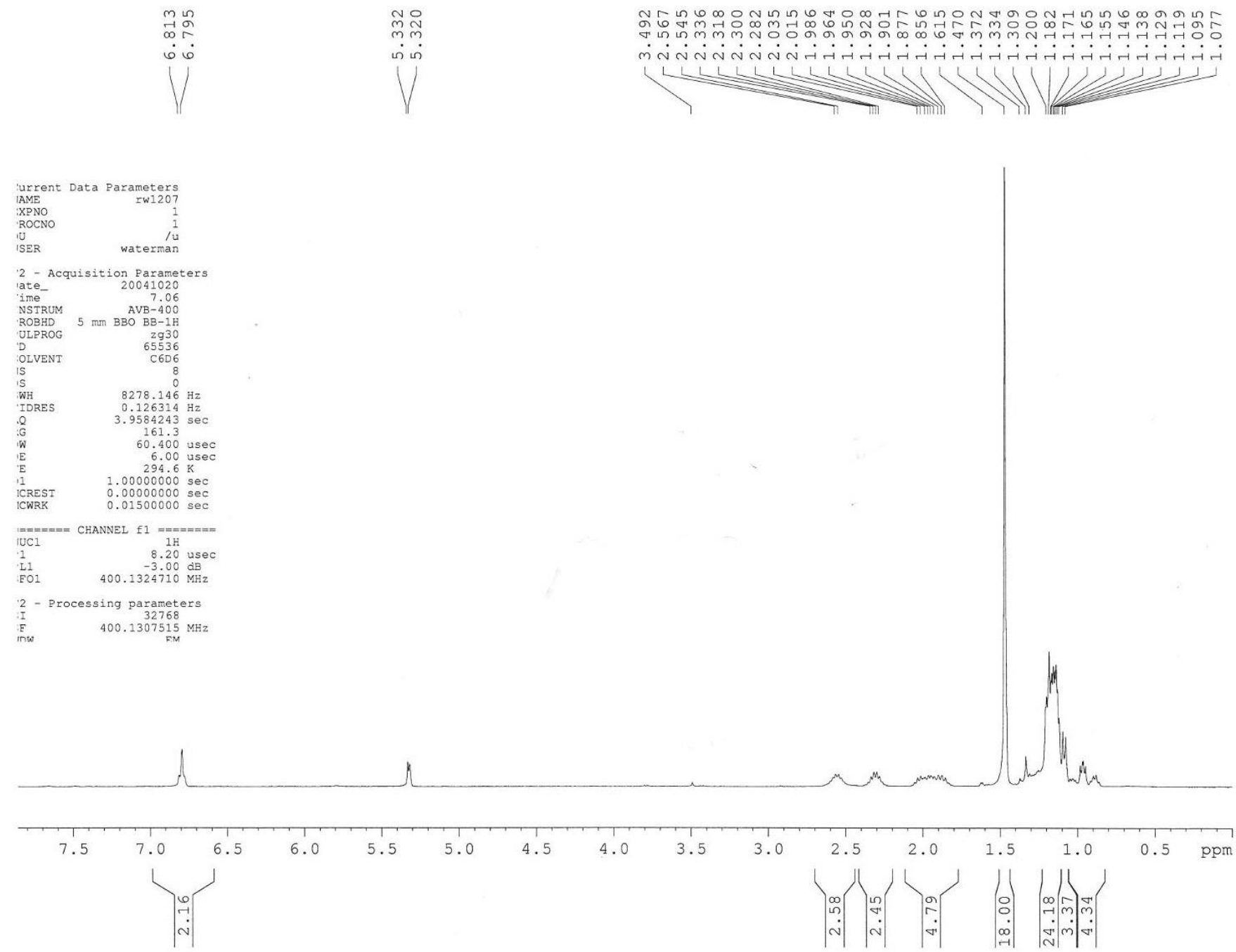

Figure S9. ${ }^{1} \mathrm{H} \mathrm{NMR}\left(\mathrm{CD}_{2} \mathrm{Cl}_{2}, 400 \mathrm{MHz}, 293 \mathrm{~K}\right)$ spectrum of 6. 


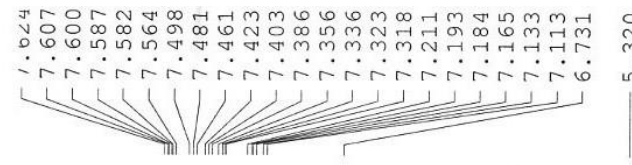

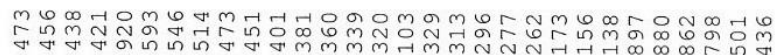
m.
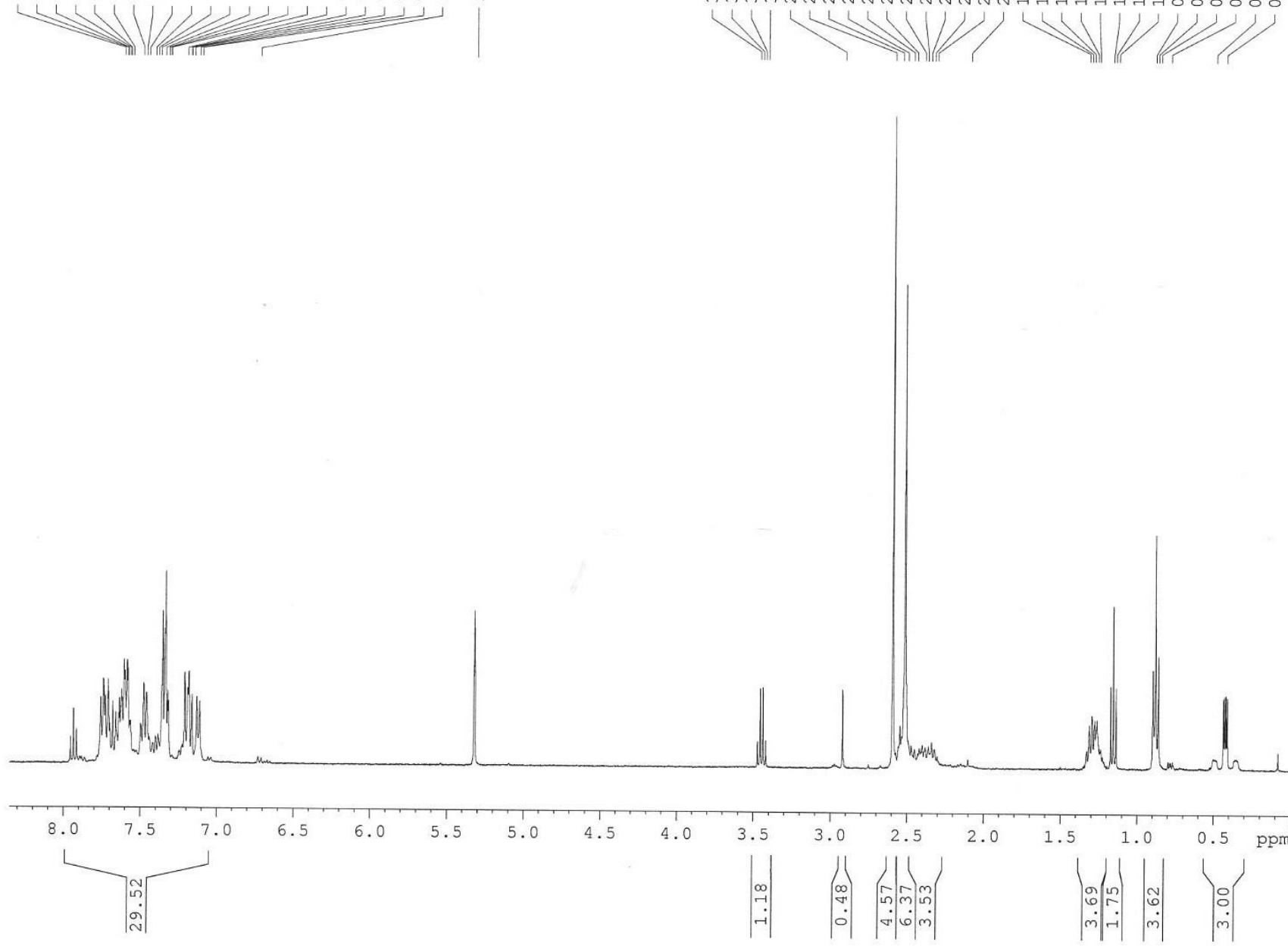

Figure S10. ${ }^{1} \mathrm{H} \mathrm{NMR}\left(\mathrm{CD}_{2} \mathrm{Cl}_{2}, 400 \mathrm{MHz}, 293 \mathrm{~K}\right)$ spectrum of 7. 


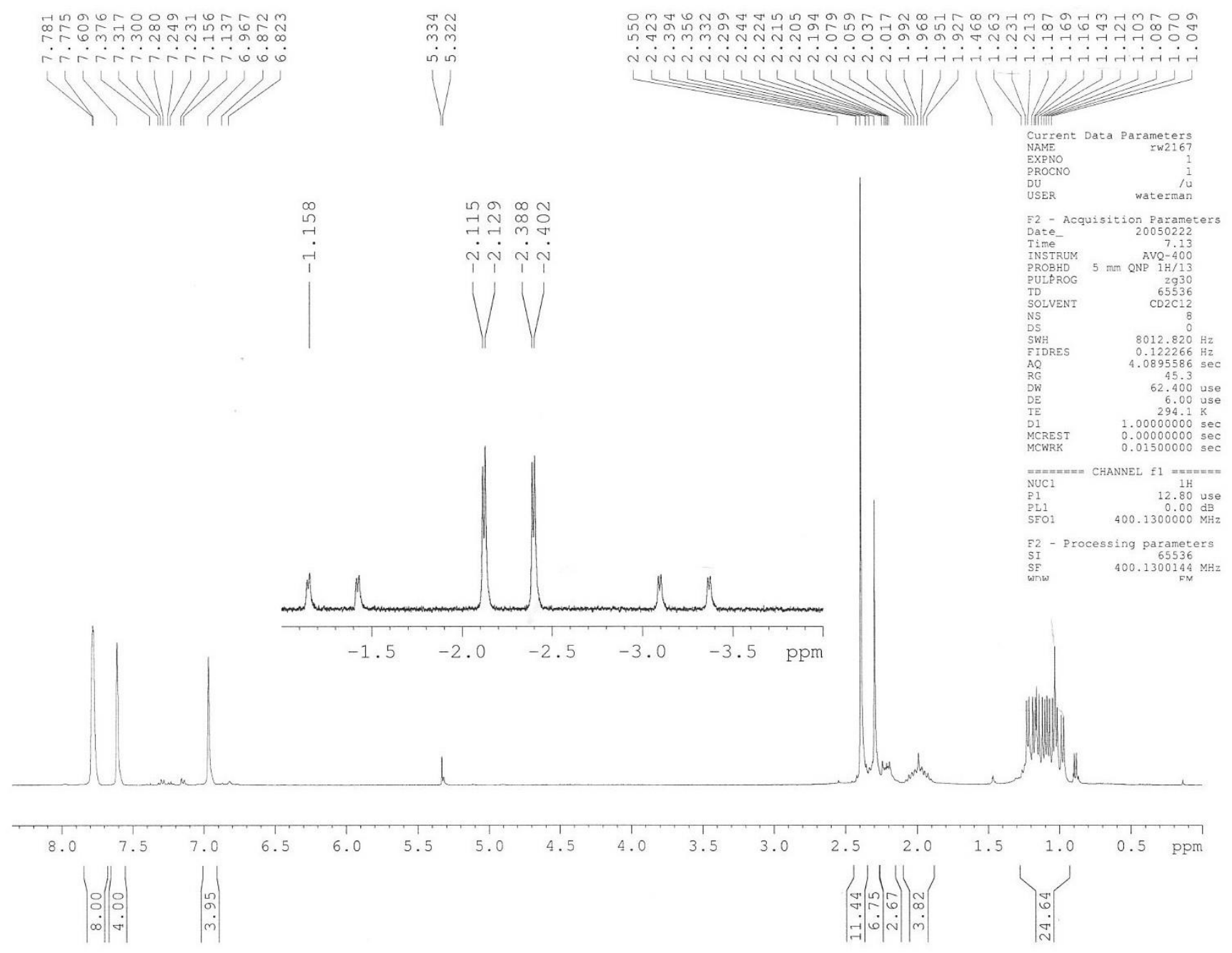

Figure S1 1. ${ }^{1} \mathrm{H} \mathrm{NMR}\left(\mathrm{CD}_{2} \mathrm{Cl}_{2}, 400 \mathrm{MHz}, 293 \mathrm{~K}\right)$ spectrum of 8 . 


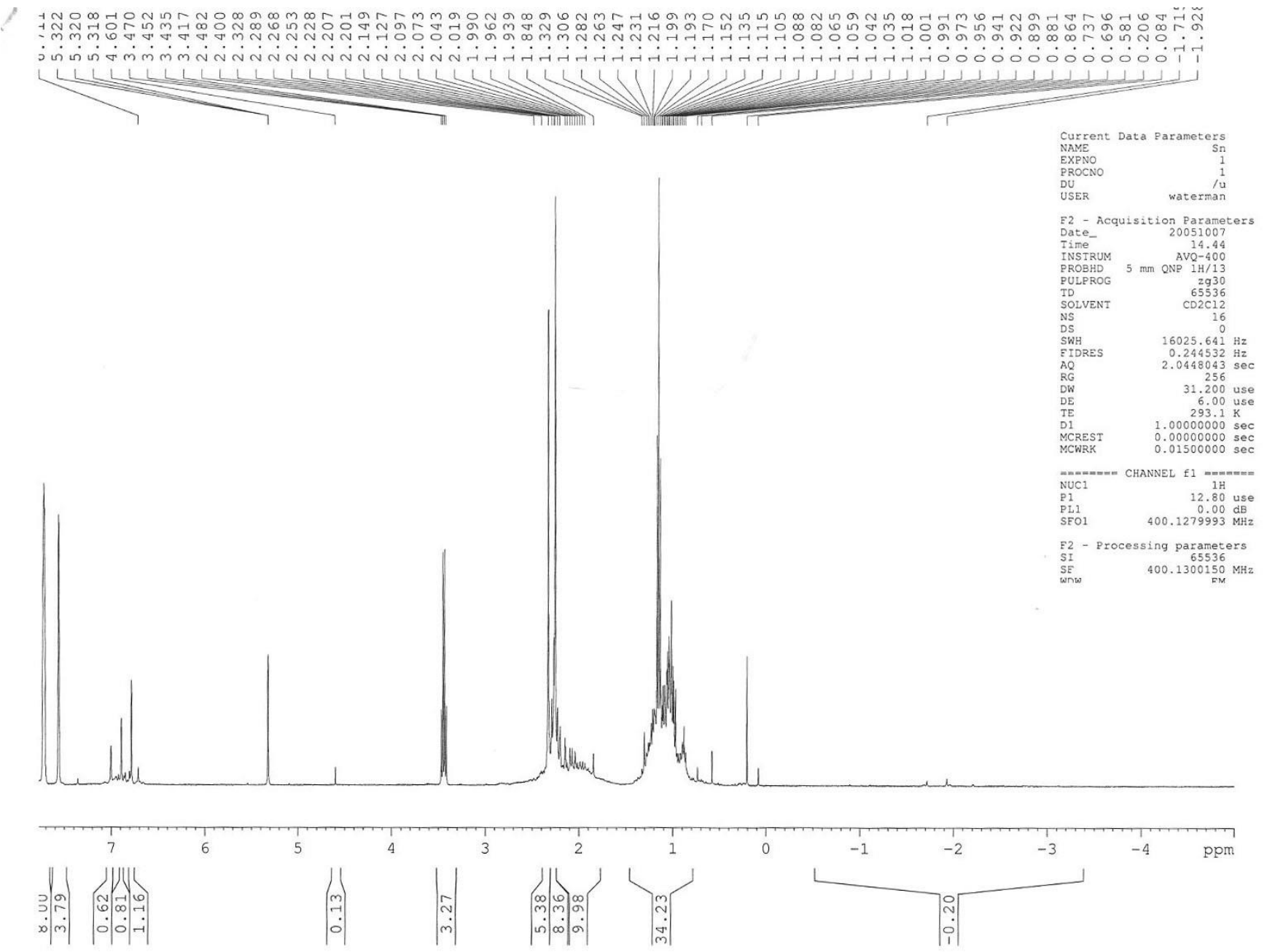

Figure S12. ${ }^{1} \mathrm{H} \mathrm{NMR}\left(\mathrm{CD}_{2} \mathrm{Cl}_{2}, 400 \mathrm{MHz}, 293 \mathrm{~K}\right)$ spectrum of 9 . 


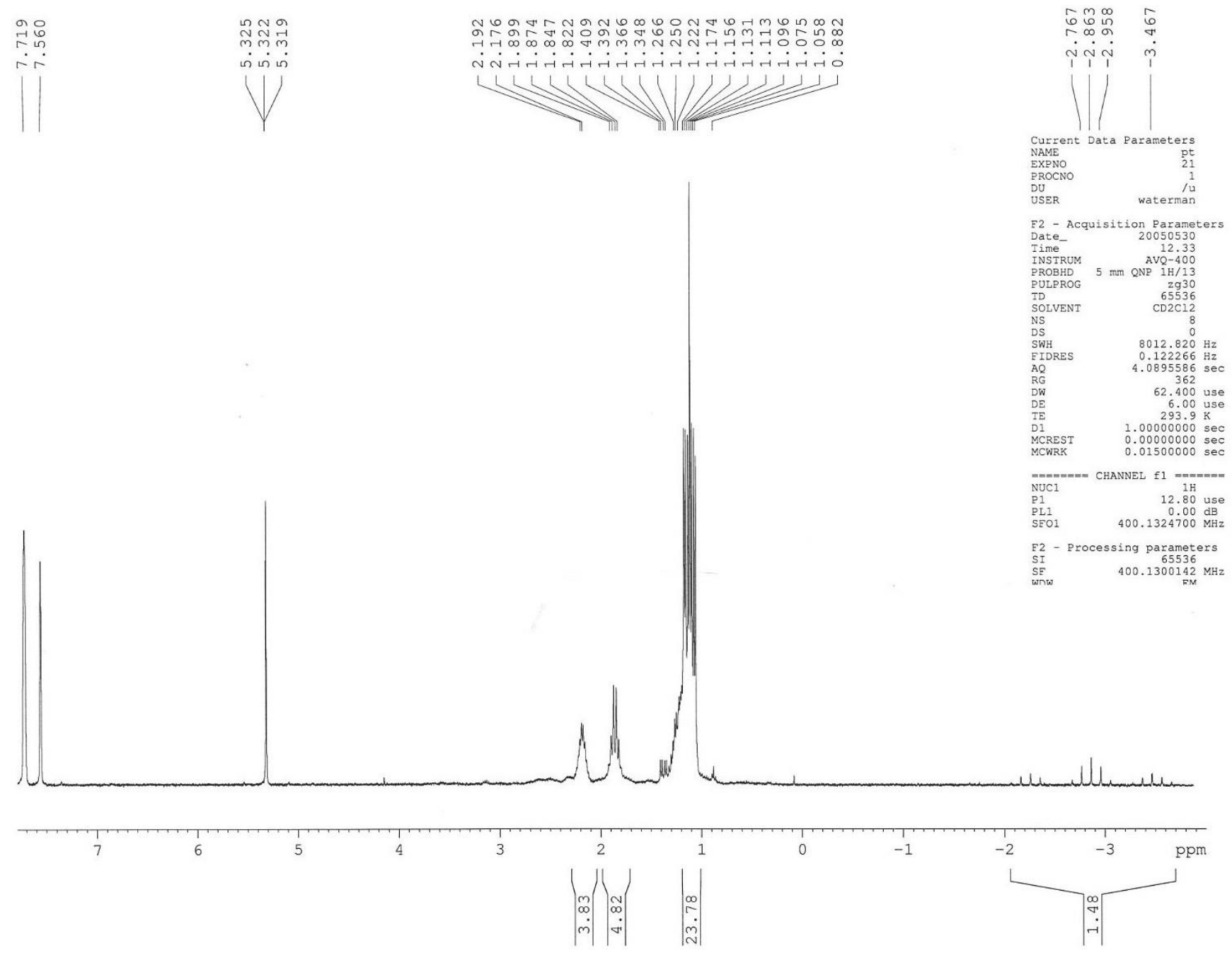

Figure S13. ${ }^{1} \mathrm{H} \mathrm{NMR}\left(\mathrm{CD}_{2} \mathrm{Cl}_{2}, 400 \mathrm{MHz}, 293 \mathrm{~K}\right)$ spectrum of 10. 


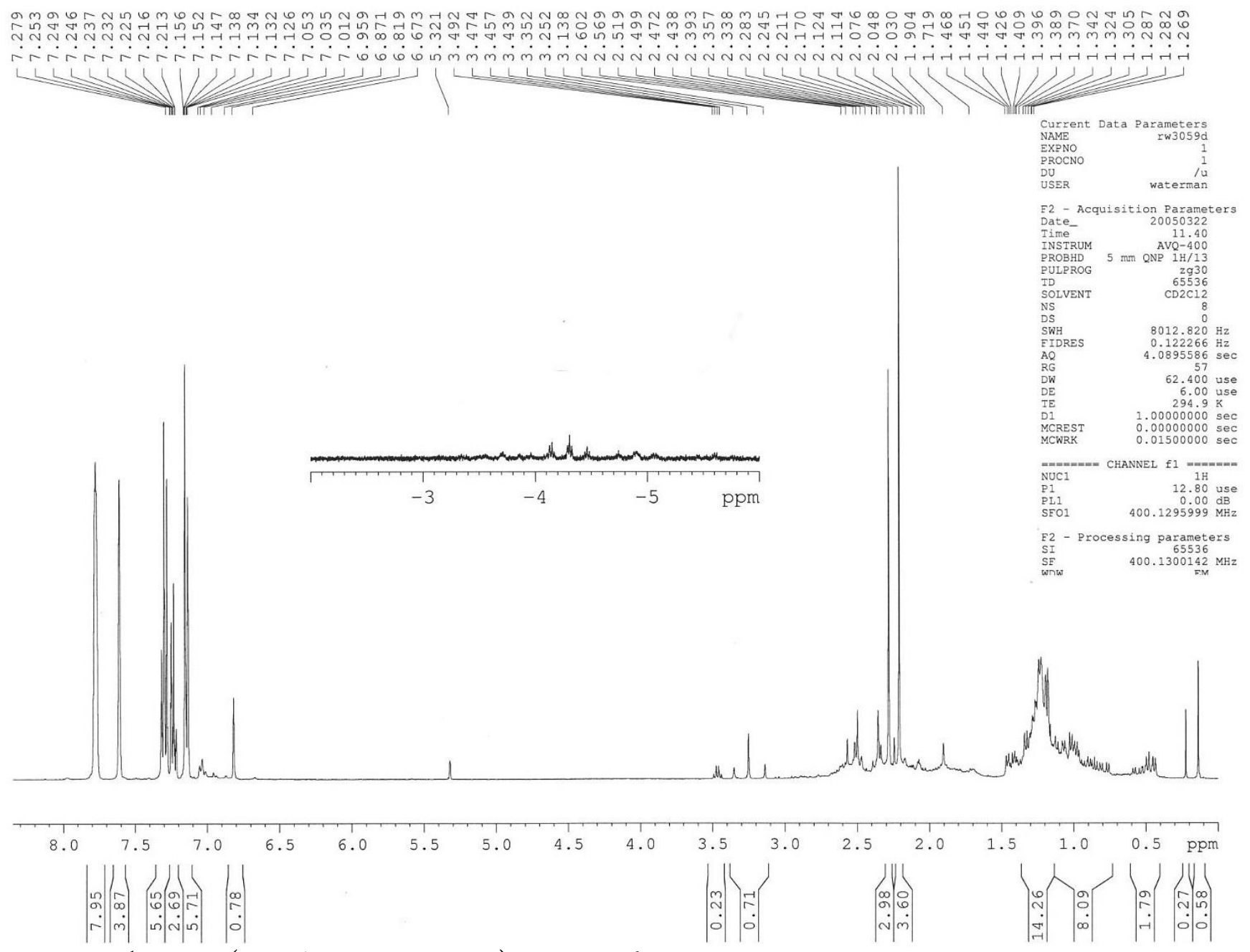

Figure S14. ${ }^{1} \mathrm{H} \mathrm{NMR}\left(\mathrm{CD}_{2} \mathrm{Cl}_{2}, 400 \mathrm{MHz}, 293 \mathrm{~K}\right)$ spectrum of 11. 


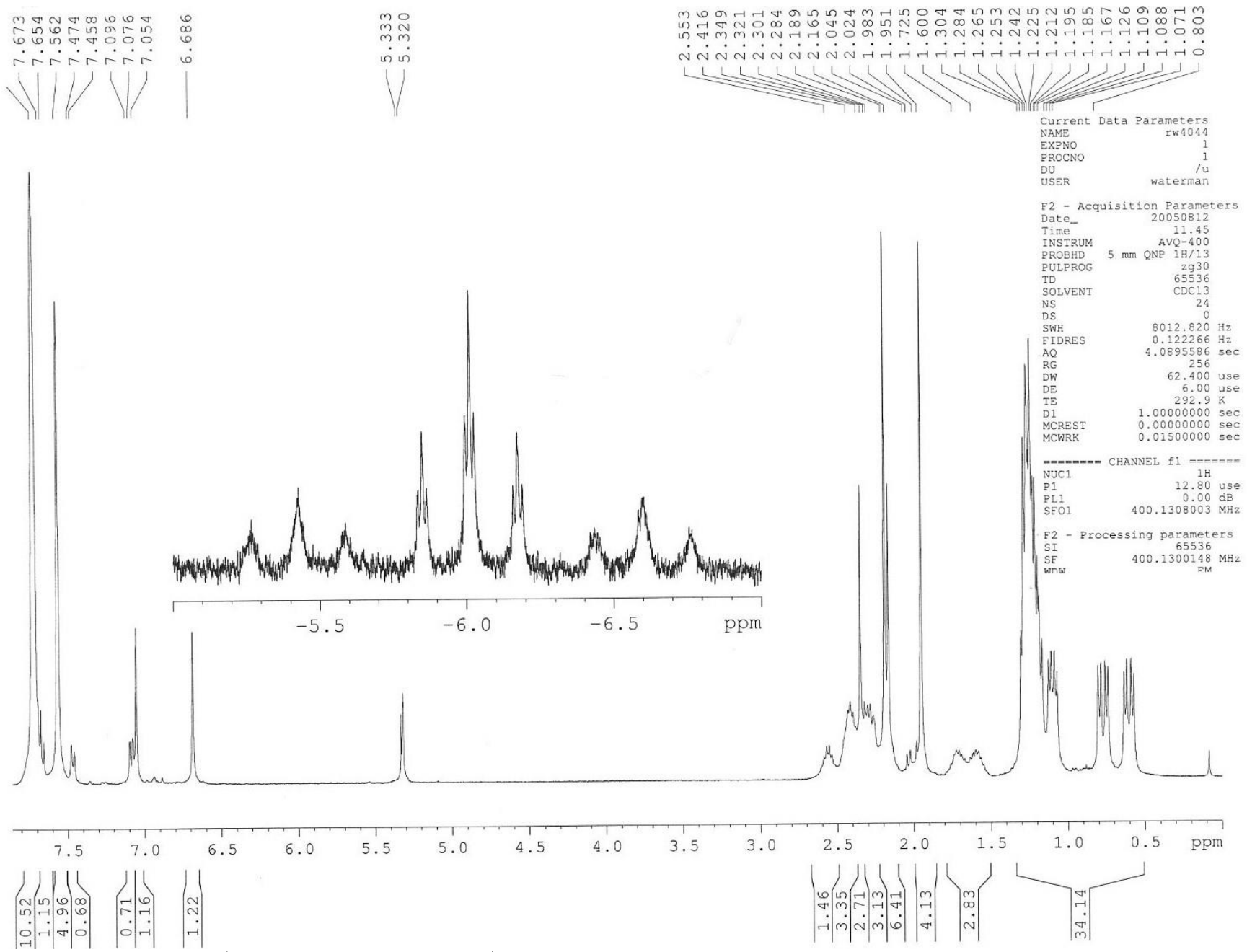

Figure S15. ${ }^{1} \mathrm{H} \mathrm{NMR}\left(\mathrm{CD}_{2} \mathrm{Cl}_{2}, 400 \mathrm{MHz}, 293 \mathrm{~K}\right)$ spectrum of 12. 


\section{REFERENCES}

(1) Sheldrick, G. M. SHELXT - Integrated Space-Group and Crystal-Structure Determination. Acta Crystallogr. Sect. Found. Adv. 2015, 71, 3-8.

(2) Sheldrick, G. M. Crystal Structure Refinement with SHELXL. Acta Crystallogr. Sect. C Struct. Chem. 2015, 71, 3-8.

(3) Dolomanov, O. V.; Bourhis, L. J.; Gildea, R. J.; Howard, J. A. K.; Puschmann, H. OLEX2 : A Complete Structure Solution, Refinement and Analysis Program. J. Appl. Crystallogr. 2009, 42, 339-341.

(4) Perdew, J. P.; Burke, K.; Ernzerhof, M. Generalized Gradient Approximation Made Simple. Phys. Rev. Lett. 1996, 77, 3865-3868.

(5) Gaussian 16, Revision A.03, Frisch, M. J.; Trucks, G. W.; Schlegel, H. B.; Scuseria, G. E.; Robb, M. A.; Cheeseman, J. R.; Scalmani, G.; Barone, V.; Petersson, G. A.; Nakatsuji, H.; Li, X.; Caricato, M.; Marenich, A. V.; Bloino, J.; Janesko, B. G.; Gomperts, R.; Mennucci, B.; Hratchian, H. P.; Ortiz, J. V.; Izmaylov, A. F.; Sonnenberg, J. L.; Williams-Young, D.; Ding, F.; Lipparini, F.; Egidi, F.; Goings, J.; Peng, B.; Petrone, A.; Henderson, T.; Ranasinghe, D.; Zakrzewski, V. G.; Gao, J.; Rega, N.; Zheng, G.; Liang, W.; Hada, M.; Ehara, M.; Toyota, K.; Fukuda, R.; Hasegawa, J.; Ishida, M.; Nakajima, T.; Honda, Y.; Kitao, O.; Nakai, H.; Vreven, T.; Throssell, K; Montgomery, J. A., Jr.; Peralta, J. E.; Ogliaro, F.; Bearpark, M. J.; Heyd, J. J.; Brothers, E. N.; Kudin, K. N.; Staroverov, V. N.; Keith, T. A.; Kobayashi, R.; Normand, J.; Raghavachari, K.; Rendell, A. P.; Burant, J. C.; Iyengar, S. S.; Tomasi, J.; Cossi, M.; Millam, J. M.; Klene, M.; Adamo, C.; Cammi, R.; Ochterski, J. W.; Martin, R. L.; Morokuma, K.; Farkas, O.; Foresman, J. B.; Fox, D. J. Gaussian, Inc., Wallingford CT, 2016.

(6) Eichkorn, K.; Weigend, F.; Treutler, O.; Ahlrichs, R. Auxiliary Basis Sets for Main Row Atoms and Transition Metals and Their Use to Approximate Coulomb Potentials. Theor Chem Acc 1997, 97, 119-124.

(7) Rappoport, D.; Furche, F. Property-Optimized Gaussian Basis Sets for Molecular Response Calculations. J. Chem. Phys. 2010, 133, 134105.

(8) Andrae, D.; Häußermann, U.; Dolg, M.; Stoll, H.; Preuß, H. Energy-Adjusted Ab Initio Pseudopotentials for the Second and Third Row Transition Elements. Theor Chem Acc 77, 123-141.

(9) Metz, B.; Stoll, H.; Dolg, M. Small-Core Multiconfiguration-Dirac-Hartree-Fock-Adjusted Pseudopotentials for Post- $d$ Main Group Elements: Application to PbH and PbO. J. Chem. Phys. 2000, 113, 2563-2569.

(10) Weigend, F. Accurate Coulomb-Fitting Basis Sets for H to Rn. Phys. Chem. Chem. Phys. 2006, 8, 1057.

(11) Humphrey, W.; Dalke, A.; Schulten, K. VMD - Visual Molecular Dynamics. J Molec Graph. 1996, 14, 33-38. 